\title{
Motor Dysfunction and Altered Synaptic Transmission at the Parallel Fiber-Purkinje Cell Synapse in Mice Lacking Potassium Channels Kv3.1 and Kv3.3
}

\author{
Hiroshi Matsukawa, ${ }^{1 \star}$ Alexander M. Wolf, ${ }^{1 \star}$ Shinichi Matsushita, ${ }^{1 \star}$ Rolf H. Joho, ${ }^{1,2}$ and Thomas Knöpfel ${ }^{1}$ \\ ${ }^{1}$ Laboratory for Neuronal Circuit Dynamics, RIKEN Brain Science Institute, Wako, Japan 351-0198, and ${ }^{2}$ Center for Basic Neuroscience, The University of \\ Texas Southwestern Medical Center, Dallas, Texas 75390-9111
}

\begin{abstract}
Mice lacking both Kv3.1 and both $\mathrm{Kv} 3.3 \mathrm{~K}^{+}$channel alleles display severe motor deficits such as tremor, myoclonus, and ataxic gait. Mice lacking one to three alleles at the Kv3.1 and Kv3.3 loci exhibit in an allele dose-dependent manner a modest degree of ataxia. Cerebellar granule cells coexpress $\mathrm{Kv} 3.1$ and $\mathrm{Kv} 3.3 \mathrm{~K}^{+}$channels and are therefore candidate neurons that might be involved in these behavioral deficits. Hence, we investigated the synaptic mechanisms of transmission in the parallel fiber-Purkinje cell system. Action potentials of parallel fibers were broader in mice lacking both Kv3.1 and both Kv3.3 alleles and in mice lacking both Kv3.1 and a single Kv3.3 allele compared with those of wild-type mice. The transmission of high-frequency trains of action potentials was only impaired at $200 \mathrm{~Hz}$ but not at $100 \mathrm{~Hz}$ in mice lacking both Kv3.1 and Kv3.3 genes. However, paired-pulse facilitation (PPF) at parallel fiber-Purkinje cell synapses was dramatically reduced in a gene dose-dependent manner in mice lacking Kv3.1 or Kv3.3 alleles. Normal PPF could be restored by reducing the extracellular $\mathrm{Ca}^{2+}$ concentration indicating that increased activity-dependent presynaptic $\mathrm{Ca}^{2+}$ influx, at least in part, caused the altered PPF in mutant mice. Induction of metabotropic glutamate receptor-mediated EPSCs was facilitated, whereas longterm depression was not impaired but rather facilitated in Kv3.1/Kv3.3 double-knockout mice. These results demonstrate the importance of Kv3 potassium channels in regulating the dynamics of synaptic transmission at the parallel fiber-Purkinje cell synapse and suggest a correlation between short-term plasticity at the parallel fiber-Purkinje cell synapse and motor performance.
\end{abstract}

Key words: cerebellar cortex; parallel fibers; Kv channels; synaptic transmission; presynaptic mechanisms; action potential repolarization; voltage-sensitive dyes

\section{Introduction}

Kv3-type voltage-gated potassium $\left(\mathrm{K}^{+}\right)$channels have high thresholds of activation and are rapidly activated during the upstroke of the action potential (AP). Therefore, Kv3.1 and Kv3.3 act as delayed rectifiers that shape the repolarization of the AP (Rudy and McBain, 2001). Mice lacking Kv3.1 and Kv3.3 potassium channels display motor deficits such as tremor and severe ataxia; in addition, they are highly ethanol sensitive (Espinosa et al., 2001). Mice lacking either the Kv3.1 or the Kv3.3 gene do not exhibit any obvious phenotypic alterations in motor function (Chan, 1997; Ho et al., 1997; Sanchez et al., 2000) Compared with several other brain regions, Kv3.1 and Kv3.3 mRNAs are present at high levels in the cerebellar cortex, and therefore, some of the motor deficits observed in Kv3.1/Kv3.3-deficient mice may result from cerebellar dysfunction. Cerebellar granule cells express both Kv3.1 and Kv3.3 (Weiser et al., 1994; Grigg et al., 2000; Li et al., 2001; Rudy and McBain, 2001), and it is possible that their similar

\footnotetext{
Received Jan. 8, 2003; revised June 23, 2003; accepted June 30, 2003.

This work was supported in part by National Institutes of Health Grant NS42210 (R.H.J.), an intramural grant from the RIKEN Brain Science Institute (T.K.), and a grant from the Myoclonus Research Foundation (R.H.J.).

*H.M., A.M.W., and S.M. contributed equally to this work.

Correspondence should be addressed to Dr. Thomas Knöpfel, Brain Science Institute, RIKEN, 2-1 Hirosawa, 3510198 Wako, Japan. E-mail: knopfe@@brain.riken.go.jp.

Copyright $\odot 2003$ Society for Neuroscience $\quad$ 0270-6474/03/237677-08\$15.00/0
}

biophysical characteristics and their coexpression in cerebellar granule cells result in functional redundancy, explaining the lack of severe phenotypic changes in mice lacking either the Kv3.1 or Kv3.3 gene. Both Kv3.1 and Kv3.3 may be involved in the repolarization phase of APs in the soma and/or in the axon of granule cells. The alteration of the repolarization of the axonal APs affects the ability for sustained high-frequency firing (Rudy and McBain, 2001) as well as the reliability and short-term plasticity at parallel fiber-Purkinje cell synapses (Sabatini and Regehr, 1997; Zucker and Regehr, 2002).

The present experiments were designed to investigate the possible correlation of motor dysfunction and alterations of the functional properties of parallel fiber-Purkinje cell synapses in mice deficient of Kv3.1 and Kv3.3 alleles. We found that lack of $\mathrm{Kv} 3.1$ and Kv3.3 alleles results in motor deficits in a gene dosedependent manner that correlate with alterations in synaptic transmission at the parallel fiber-Purkinje cell synapses.

\section{Materials and Methods}

Breeding of Kv3.1- and Kv3.3-deficient mice. The generation, initial characterization, and genotyping of mutant mice lacking Kv3.1 and $\mathrm{Kv} 3.3 \mathrm{~K}^{+}$ channel genes have been described (Espinosa et al., 2001). Mice were kept on a $12 \mathrm{hr}$ light/dark cycle. Experiments using the force actometer were performed with male and female mice $(\sim 3$ months of age $)$ on a mixed genetic background of $129 / \mathrm{Sv} \times \mathrm{C} 57 \mathrm{BL} / 6 \times$ ICR. Most experiments 
A

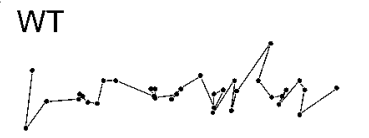

SKO

TM

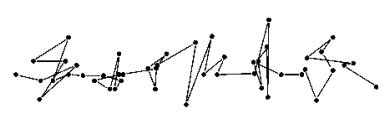

DKO

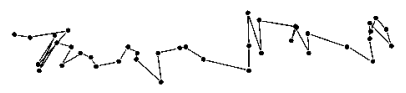

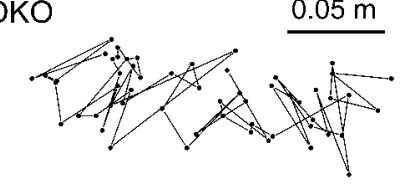

B

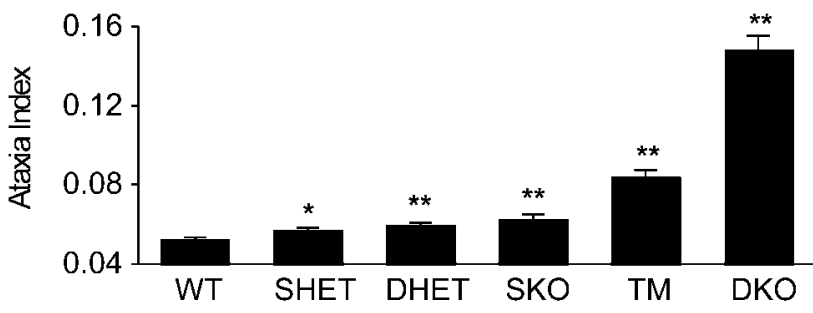

Figure 1. Walking trajectories of mice lacking Kv3.1 or Kv3.1 alleles. A, Walking trajectories of a WT control mouse (Kv3. $1^{+/+} \mathrm{Kv3} .3^{+/+}$), a Kv3.1 single mutant (SKO) (Kv3.1 ${ }^{-/-} \mathrm{Kv3} 3.3^{+/+}$), a mouse lacking both Kv3.1 alleles and one Kv3.3 allele (TM) (Kv3.1 ${ }^{-1-} \mathrm{Kv} 3.3^{+/-}$), and a mouse lacking all of the Kv3.1 and Kv3.3 alleles (DKO) (Kv3.1 ${ }^{-1-} \mathrm{Kv} 3.3^{-1-}$ ). Dots indicate center of force of the mouse measured at $50 \mathrm{~Hz}$ and connected by lines. Note increased fluctuations perpendicular to the main direction of movement with increasing lack of Kv3.1 and Kv3.3 alleles. B, Ataxia indices obtained from mice lacking varying numbers of functional Kv3.1 and Kv3.3 alleles. Error bars indicate mean \pm SEM values obtained from $21 \mathrm{WT}, 30 \mathrm{Kv} 3.1^{+/-} \mathrm{Kv} 3.3^{+/+}$(SHET), $14 \mathrm{Kv3} .1^{+/-} \mathrm{Kv3} 3.3^{+/-}$(DHET), 7 SKO, 12 TM, and 5 DKO mice. Asterisks indicate statistical significant differences from WT $\left({ }^{*} p<\right.$ $\left.0.05 ; *{ }^{* *} p 0.01\right)$.

involving $\mathrm{Kv} 3.1^{+/+} \mathrm{Kv} 3.3^{+/+}$[wild-type (WT)] mice, Kv3.1 $1^{+/-}$ $\mathrm{Kv} 3.3^{+1-}$ mice [double heterozygotes (DHETs)], and $\mathrm{Kv} 3.1^{-1-}$ $\mathrm{Kv} 3.3^{-1-}$ mice (double homozygotes [double knock-outs (DKOs)]) were performed with offspring from DHET $\times$ DHET mating to equalize the influence of unavoidable genetic variability among individual mice of different $\mathrm{Kv} 3.1 / \mathrm{Kv} 3.3$ genotypes. Kv3.1 ${ }^{+/-} \mathrm{Kv} 3.3^{+/+}$mice [single heterozygotes (SHETs)], Kv3.1 $1^{-/-} \mathrm{Kv} 3.3^{+/+}$mice [single knock-outs (SKOs)], and Kv3.1 ${ }^{-1-} \mathrm{Kv} 3.3^{+/-}$[triple-minus (TM)] mice were generated using SHET mice that had been obtained by recombination in DHET mating pairs.

Measurement of walking trajectories. Mice were placed for $6 \mathrm{~min}$ on a $28 \times 28 \mathrm{~cm}$ platform of a force-plate actometer that allows the determination of the animal's center of force (COF) with high spatial $(<1 \mathrm{~mm})$ and temporal $(20 \mathrm{msec})$ resolution (Fowler et al., 2001). The COF (approximately equivalent to the position of the mouse) was monitored (sampled at $50 \mathrm{~Hz}$ ) while the mouse was allowed to walk and explore the environment. For the purpose of data analysis, walking was defined as movement when COFs measured $400 \mathrm{msec}$ before and $400 \mathrm{msec}$ after a given time point were at a distance of $>6 \mathrm{~cm}$ (corresponding to a walking velocity of $>15 \mathrm{~cm} / \mathrm{sec}$ ). During periods of walking, the triangular surface areas defined by three consecutive COF measurements and the walking distances defined by the COFs were calculated and summed. The ataxia index was calculated as the total surface area divided by the total distance traveled. The value of this ataxia index is zero if the walking trajectory is a straight line and increases with increasing deviation of the COF from the main movement direction.

Voltage-sensitive dye recordings. Slices of the surface of the cerebellar cortex were obtained from adult ( $40-60 \mathrm{~d}$ of age) mice. Animals were deeply anesthetized with ether and decapitated. The cerebellar vermis was removed and placed in ice-cold artificial CSF (ACSF) containing (in $\mathrm{mm}$ ): $125 \mathrm{NaCl}, 25 \mathrm{NaHCO}_{3}, 10$ glucose, $3 \mathrm{KCl}, 2 \mathrm{CaCl}_{2}, 1 \mathrm{MgSO}_{4}$, and 1 $\mathrm{NaH}_{2} \mathrm{PO}_{4}$, oxygenated with $95 \% \mathrm{O}_{2}-5 \% \mathrm{CO}_{2}$. Superficial slices of the cerebellar vermis, coplanar to the cerebellar surface, were cut of lobules 6/7 (maximum thickness, 400-500 $\mu \mathrm{m}$ ) with a vibroslicer (VT1000S; Leica, Nussloch, Germany), left to recover at $35^{\circ} \mathrm{C}$ for $>30 \mathrm{~min}$, and subsequently stored at room temperature $\left(24^{\circ} \mathrm{C}\right)$. Slices were stained with the voltage-sensitive dye di-4-ANEPPS [Molecular Probes, Eugene, OR; $5 \mu \mathrm{M}$ prepared from a stock solution in ethanol/DMSO (2:1), diluted 200:1 in oxygenated ACSF] for $30 \mathrm{~min}$ and transferred to a recording chamber. Slices were continuously superfused with oxygenated ACSF containing bicuculline methiodide ( $20 \mu \mathrm{M}$; Tocris, Bristol, UK) and 2,3dioxo-6-nitro-7-sulfamoylbenzo(f)quinoxaline (NBQX) $(20 \mu \mathrm{M}$; Tocris) at a rate of $2 \mathrm{ml} / \mathrm{min}$. All of the measurements were made at $23-25^{\circ} \mathrm{C}$. An epifluorescence setup consisting of a $1.6 \times$ objective, a dichroic mirror $(575 \mathrm{~nm})$, a long-pass filter $(590 \mathrm{~nm})$, and an inverted $1.6 \times$ projection objective was mounted above the slice. Fluorescence was excited with a laser (532 nm; Verdi; Coherent, Palo Alto, CA) and detected with a high-speed CCD camera (FastOne; Pixel Vision, Tigard, OR) at $2.7 \mathrm{kHz}$ image rate. Current pulses $(200 \mu \mathrm{sec} ; 40-70 \mu \mathrm{A})$ were delivered to the surface of the molecular layer through a monopolar platin-iridium electrode $(0.5 \mathrm{M} \Omega)$. Optical responses are the average of 16 trials repeated at $0.05 \mathrm{~Hz}$.

Calculation of action potential shape parameters. Series of fluorescence images were analyzed using IDL 5.2. Fluorescence images were filtered with a $3 \times 3(42 \times 42 \mu \mathrm{m})$ box filter. Baseline level of fluorescence was taken as the average of eight fluorescence time points, four preceding stimulation by $0.74-1.85 \mathrm{msec}$ and four $8.51-9.62 \mathrm{msec}$ after stimulation. The AP maximum was defined as the peak fluorescence decrease. Time points of half-maximal AP rise and fall were calculated using linear interpolation between the fluorescence measurement above and below half-maximum. AP width (see Fig. $2 C, D$, width at half-maximum) was the difference between rise time point and fall time point. The AP shape (see Fig. 2E) was obtained by aligning the fluorescence time courses obtained from individual pixels relative to their rise time point and binning the resulting time course at $5 \mathrm{kHz}$.

High-frequency action potential firing. Fluorescence time courses (see Fig. $3 A, C$ ) were obtained by subtracting a baseline (4.44 msec moving lower quartile) from the raw fluorescence measurements to remove slow components. For comparison (see Fig. $3 B, D$ ), peak heights were normalized relative to the maximum peak height.

Whole-cell recording. Parasagittal cerebellar slices (230-250 $\mu \mathrm{m})$ were prepared from 19- to 26-d-old mice following previously established techniques (Reichelt and Knöpfel, 2002). After recovery ( $>1 \mathrm{hr}$ ) at 23$25^{\circ} \mathrm{C}$, slices were continuously perfused at $2 \mathrm{ml} / \mathrm{min}$ with ACSF containing $20 \mu \mathrm{M}$ bicuculline methiodide (see above). Whole-cell recordings were obtained from Purkinje cells using borosilicate glass pipettes (3-4.5 $\mathrm{M} \Omega$ ). Purkinje cells were voltage clamped at $-65 \mathrm{mV}$, and series resistances were compensated up to $80-90 \%$ using a Axopatch $200 \mathrm{~B}$ amplifier (Axon Instruments, Foster City, CA). The internal solution contained (in mM): $120 \mathrm{~K}$-gluconate, $9 \mathrm{KCl}, 10 \mathrm{KOH}, 4 \mathrm{NaCl}, 10$ HEPES, 3.48 $\mathrm{MgCl}_{2}, 0$ or 0.05 EGTA, 17.5 sucrose, $4 \mathrm{Na}_{2}$-ATP, and $0.4 \mathrm{Na}_{3}$-GTP, adjusted to $\mathrm{pH} 7.3$ using $\mathrm{KOH}$. For electrical stimulation of parallel fibers, negative current pulses (500 $\mu \mathrm{sec} ; 2-30 \mu \mathrm{A})$ were delivered through a glass pipette (filled with external solution) positioned in the molecular layer. Paired-pulse facilitation (PPF) was induced every $30 \mathrm{sec}$ and test responses for LTD were recorded at $0.1 \mathrm{~Hz}$. Pairing protocols for testing for the occurrence of parallel-fiber synaptic plasticity were conducted in voltage-clamp mode. In experiments involving variation of extracellular $\mathrm{Ca}^{2+}$ concentration, the sum of $\mathrm{Mg}^{2+}$ and $\mathrm{Ca}^{2+}$ concentration was kept constant at $4 \mathrm{~mm}$.

Chemicals and drugs. Unless noted otherwise, all of the chemicals were obtained from Sigma (St. Louis, MO).

Statistical analysis. Values obtained from different experimental groups were tested for statistical differences using $t$ test under Origin software (OriginLab, Northampton, MA).

\section{Results}

Mice lacking Kv3.1/Kv3.3 genes are ataxic

Previous work showed that mice lacking both Kv3.1 and both Kv3.3 alleles (DKO mice; $-/-,-/-$ ) exhibit severe ataxia, myoclonus, and tremor (Espinosa et al., 2001). In contrast, mice lacking a single Kv3.1 and a single Kv3.3 allele (DHET mice; + /-, $+/-)$ and both single mutants $(-/-,+/+$; or $+/+,-/-)$ did not exhibit obvious phenotypic alterations in motor behavior 
A
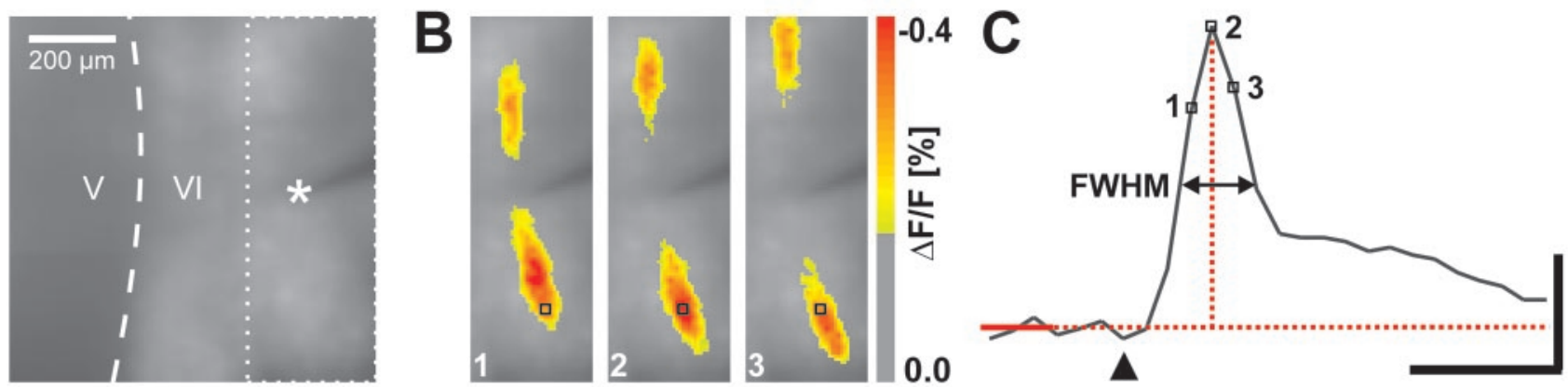

D
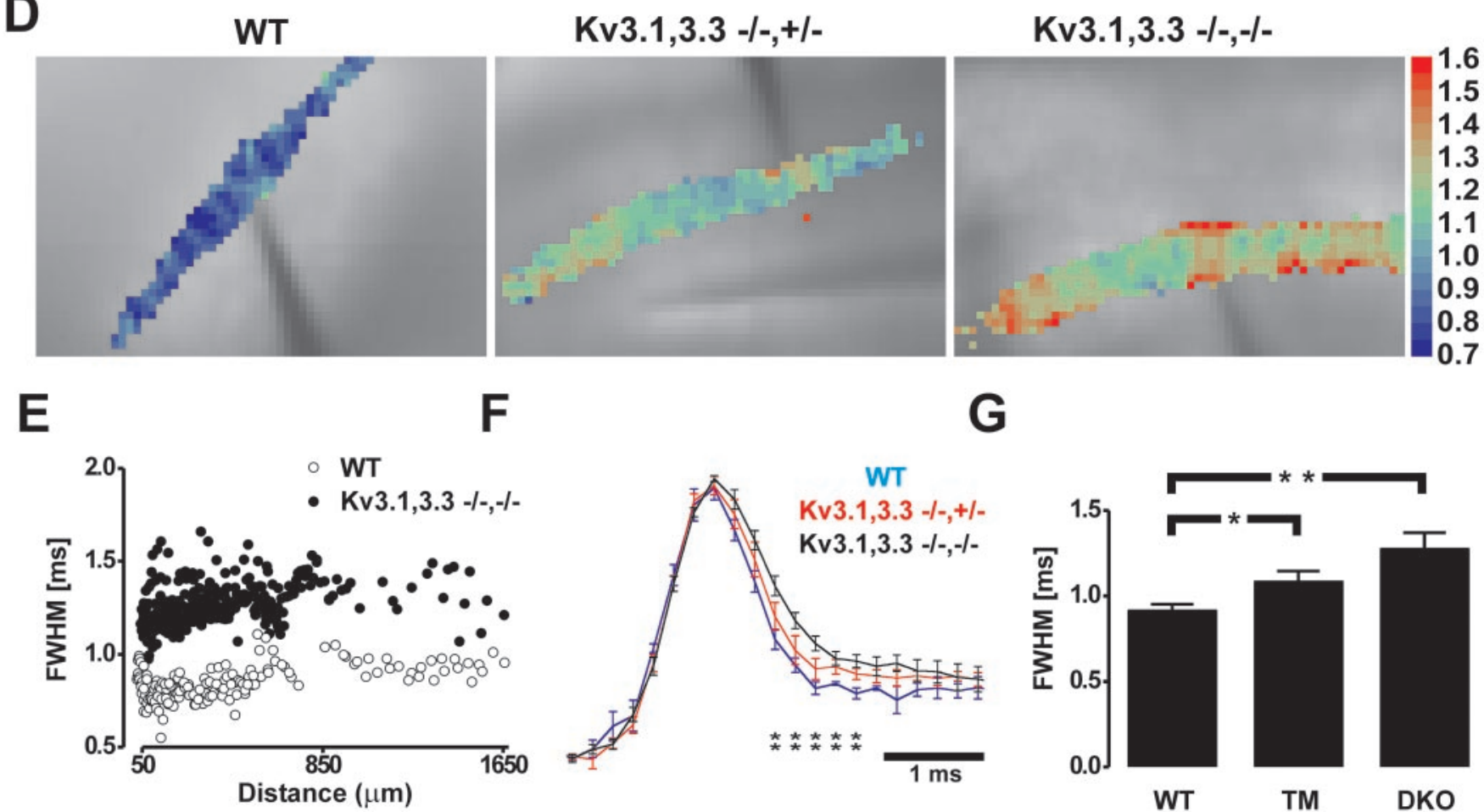

F

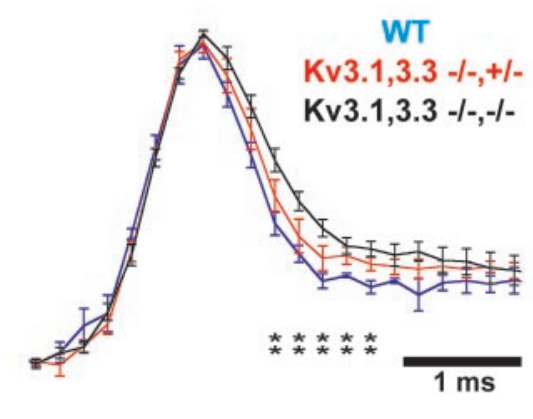

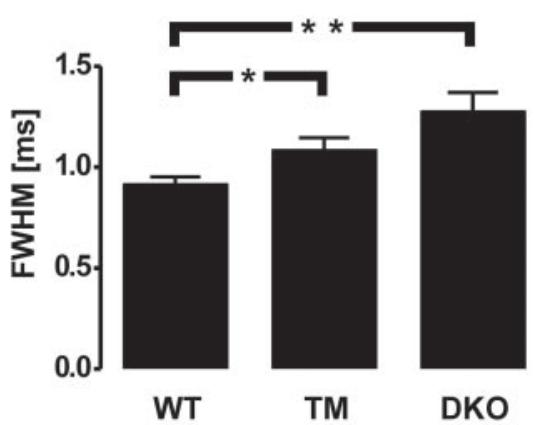

Figure 2. Parallel-fiber action potential shape is affected by lack of Kv3.1 and Kv3.3 alleles. $A$, Fluorescence image of the lobule V/VI cerebellar surface stained with the voltage-sensitive dye. The stimulation electrode (asterisk) was placed in lobule VI. $B$, Color-coded fluorescence change representing depolarization 1.11 ( 1 ), 1.48 (2), and 1.85 (3) msec after stimulation in area outlined by dotted line in $A$. Fluorescence change $(\Delta F)$ is expressed as percentage of baseline fluorescence $(F)$. C, Fluorescence time course at the point marked by a black square in $B$. Width of compound action potential is measured at half-maximum fluorescence change relative to a baseline. The stimulation time point is marked by a black triangle. Calibration: 2 msec, $0.2 \%$. Data shown in $A-C$ were obtained from a WT mouse. D, Color-coded maps of action potential width obtained from a WT mouse, a TM mouse, and a DKO mouse. E, Action potential width plotted against distance from the stimulation electrode in a WT preparation and a DKO preparation. Note small increase in the width of the measured compound action potential with increasing distance from the stimulation site, indicating some inhomogeneity in AP conduction velocities of individual action potentials in both genotypes. $F$, Mean \pm SEM action potential shape (resampled at $5 \mathrm{kHz}$ ( Methods). G, Mean \pm SEM action potential width for WT(917 $\pm 36 \mu$ sec; $n=7)$, TM (1088 $\pm 56 \mu$ sec; $n=6)$, and DKO (1280 $\pm 89 \mu$ sec; $n=7)$. FWHM indicates fluorescence width at half-maximum. Asterisks indicate significant differences from WT $\left({ }^{*} p<0.05 ;{ }^{* *} p<0.01\right)$.

(Ho et al., 1997; Sanchez et al., 2000; Espinosa et al., 2001). In this study, we took advantage of a highly sensitive device that allows measurement of the COF of freely moving mice at a spatial resolution of $<1 \mathrm{~mm}$ and a temporal resolution of $20 \mathrm{msec}$ (Fowler et al., 2001). At this resolution, the trajectory of the COF during walking deviated clearly from a straight line even in normal control mice (Fig. 1A). These deviations increased with increasing lack of Kv3.1 and Kv3.3 alleles (Fig. 1A). The ataxia index (see Materials and Methods) was calculated for six different genotypes with respect to the lack of Kv3.1 and Kv3.3 alleles (Fig. $1 B$ ). The ataxia index increased with increasing lack of Kv3.1 and Kv3.3 alleles. It appeared to be slightly yet significantly $(p<0.05)$ different from WT already with the lack of one Kv3.1 allele (Fig. $1 B$ ). Notably, mice lacking both Kv3.1 alleles and one Kv3.3 allele (TM mice; $-/-,+/-)$ and DKO mice exhibited an ataxic gait that could be easily revealed by simple observation of the animals.

\section{Duration of parallel-fiber action potential is altered in}

Kv3.1/Kv3.3-deficient mice

The observation that motor dysfunction (severe ataxia, myoclonus, and tremor) was only severely impaired when both Kv3.1 and Kv3.3 channels were absent suggests functional redundancy of these two channels. Given the known expression pattern of Kv3.1 and Kv3.3 channels (Weiser et al., 1994; Grigg et al., 2000; Li et al., 2001; Rudy and McBain, 2001), cerebellar granule cells are the most obvious candidate neurons that coexpress Kv3.1 and $\mathrm{Kv} 3.3$ and are involved in motor function. The Kv3.1 and Kv3.3 channels might be involved in repolarization of the axonal AP of 
granule cells (the parallel fibers). To examine this possibility, we measured the shape of compound parallel-fiber APs by means of voltage-sensitive dyes in WT, TM, and DKO mice. We used cerebellar slices cut coplanar with the surface of cerebellar lobules 6/7 (Fig. 2A). This preparation allows for optical imaging of parallel fibers over their full trajectory (Vranesic et al., 1994). Synaptic transmission was blocked with NBQX and bicuculline $(20 \mu \mathrm{M})$, and a high-speed CCD camera was used to record the propagating compound AP (Fig. 2B).

Electrical stimulation of the WT cerebellar surface induced compound APs that propagated in both transverse directions (Fig. 2B) (Vranesic et al., 1994). The width of the compound AP was measured from the averaged fluorescence time course of individual responsive pixels (Fig. 2C). The optically recorded compound action potential exhibits an apparent afterdepolarization. This signal component has been described previously, and it is unclear whether it represents depolarization of glial cells or a spike afterdepolarization of the parallel fiber (Vranesic et al., 1994; Sabatini and Regehr, 1997). Propagating APs were also observed in mutant mice as illustrated in Figure 2D, which shows a color-coded map of AP width for representative WT, TM, and DKO brain slices. Plotting the width of the AP obtained from individual responsive pixels against the distance from the site of stimulation revealed that the measured width slightly increased with increasing distance (Fig. 2E). This observation is consistent with previous data obtained with similar methods showing some inhomogeneity in action potential propagation velocity of parallel fibers (Vranesic et al., 1994). This slight dispersion of the compound action potential is, however, much smaller than the differences seen between AP width of WT, TM, and DKO mice (Fig. 2D). The average AP shape (Fig. $2 F$ ) shows that the AP of WT and Kv3.1/Kv3.3-deficient mice significantly differed during the repolarizing phase. The AP width was significantly larger in DKO mice (mean \pm SEM, $1280 \pm 89 \mu \mathrm{sec}$; $n=7 ; p<0.01)$ and TM mice $(1088 \pm 56 \mu$ sec; $n=6 ; p<0.05)$ compared with that of WT mice $(917 \pm 36 \mu$ sec; $n=7)$ (Fig. $2 G$ ).

\section{High-frequency firing is impaired in parallel fibers of} Kv3.1/Kv3.3-deficient mice

Because Kv3-type potassium channels are involved in sustained high-frequency firing (Rudy and McBain, 2001; Porcello et al., 2002; Macica et al., 2003), we examined whether the ability of granule cell axons to follow sustained high-frequency stimulation was affected. Figure $3, A$ and $B$, shows that both DKO and WT parallel fibers faithfully conduct APs at $100 \mathrm{~Hz}$ with only a small amplitude reduction in DKO relative to $\mathrm{WT}$ at the end of the train of 10 pulses. Only when stimulated at $200 \mathrm{~Hz}$ (Fig. 3C,D) is it apparent that the DKO axons cannot sustain this firing rate. WT axons reliably conduct APs even at $200 \mathrm{~Hz}$ with relatively little reduction in amplitude, whereas in the $\mathrm{DKO}$, conduction failures are close to $80 \%$ for the last of 20 pulses at $200 \mathrm{~Hz}$.
Altered short-term plasticity at the parallel fiber-Purkinje cell synapse in mice deficient of Kv3.1 and Kv3.3 alleles Rapid repolarization of APs is not only required for sustained high-frequency firing but also limits the activity-dependent influx of calcium into presynaptic specialization (Sabatini and Regehr, 1997) and, thereby, synaptic dynamics and plasticity (Zucker and Regehr, 2002). To investigate whether lack of Kv3.1 and Kv3.3 channels affects synaptic properties, we compared PPF at the parallel fiber-Purkinje cell synapse between WT mice and mice lacking different numbers of Kv3.1 and Kv3.3 alleles (Fig. 4). The degree of PPF observed in DKO mice was dramatically reduced compared with that of wild-type mice. An intermediate degree of PPF was observed in SHET $(-/+,+/+)$, SKO $(-/-$, $+/+)$, DHET $(+/-,+/-)$, and TM $(-/-,+/-)$ mice. The degree of PPF closely correlated with the number of intact Kv3.1 and Kv3.3 alleles (Fig. 4B).

Altered PPF at the parallel fiber-Purkinje cell synapse can be reversed by decreasing extracellular calcium concentration We hypothesized that prolonged APs resulted in decreased PPF because of increased activity-dependent presynaptic calcium influx. This assumption is based on the residual calcium concept (Zucker and Regehr, 2002). Alternatively, altered PPF might result from altered release of $\mathrm{K}^{+}$(Malenka et al., 1981) or adaptive changes in the mutant mice. To test directly the hypothesis that decreased PPF in mutant mice resulted from increased presynaptic calcium influx, the dependence of PPF on the extracellular calcium concentration was measured. EPSCs induced by pairs of parallel-fiber stimuli at varying interstimulus intervals were recorded at different concentrations of extracellular $\mathrm{Ca}^{2+}$ $\left(\left[\mathrm{Ca}^{2+}\right]_{\mathrm{o}}\right)$. The concentration of $\mathrm{Mg}^{2+}$ in the extracellular solution was also adjusted such that the total concentration of divalent cations was constant at $4 \mathrm{~mm}$. In wild-type and mutant mice, PPF decreased with increasing $\left[\mathrm{Ca}^{2+}\right]_{0}$; however, comparable 
A
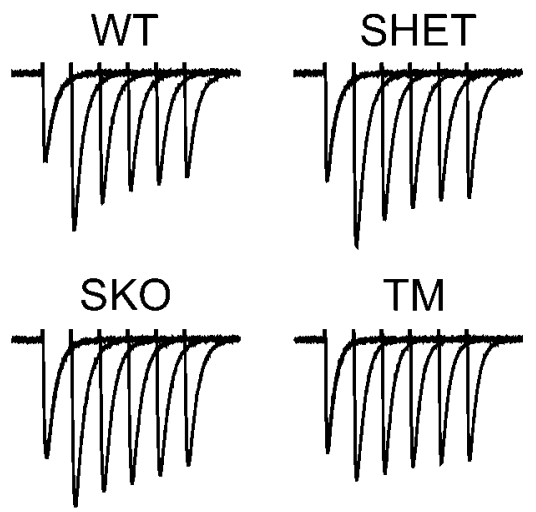

B
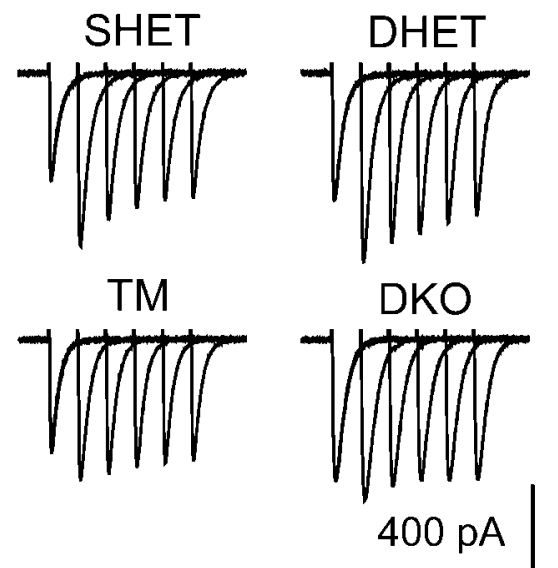

$100 \mathrm{~ms}$

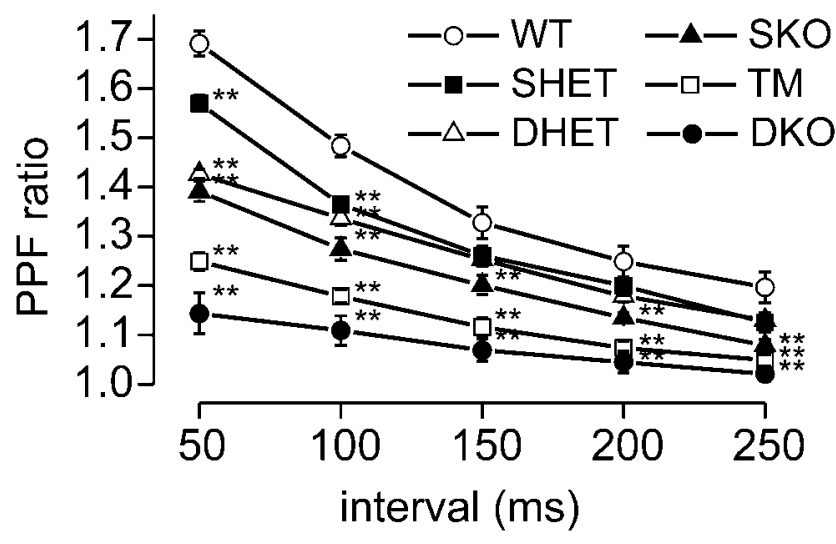

Figure 4. Short-term plasticity is altered in mice lacking Kv3.1 and Kv3.3 alleles. $A$, Purkinje cell EPSC PPF at intervals of 50, 100, 150, 200, and 250 msec in Kv3.1 $1^{+/+} \mathrm{Kv3} .3^{+/+}$(WT),

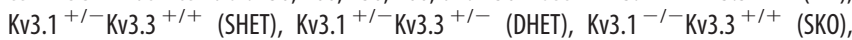
$\mathrm{Kv} 3.1^{-1-} \mathrm{Kv} 3.3^{+/-}$(TM), and Kv3.1 $1^{-1-} \mathrm{Kv} 3.3^{-1-}$ (DKO) mice. Stimulus artifacts were suppressed for clarity. $B$, PPF ratio values (mean \pm SEM; $n=6$ cells for each category) plotted against paired-pulse interval. Asterisks indicate significant differences $(p<0.01)$ from WT. Note the gradual reduction of PPF with reduction of functional Kv3.1 and Kv3.3 alleles.

PPF values were obtained at lower $\left[\mathrm{Ca}^{2+}\right]_{\mathrm{o}}$ in DKO than in WT mice (Fig. 5, Table 1). Thus, a similar degree of PPF was obtained for DKO in $0.5-1 \mathrm{mM} \mathrm{Ca}^{2+}$ and for WT in $2 \mathrm{mM} \mathrm{Ca}^{2+}$. Thus, the decreased PPF in mutant mice could be reversed by lowering $\left[\mathrm{Ca}^{2+}\right]_{0}$, demonstrating that the alteration in PPF could be accounted for, at least in part, by increased activity-dependent presynaptic calcium influx.

\section{Application of low concentrations of TEA qualitatively, but not quantitatively, replicates the altered PPF of DKO mice in WT mice}

Previous work by Sabatini and Regehr (1997) showed that application of the potassium channel blocker TEA results in a broadening of parallel-fiber AP and greatly enhanced postsynaptic currents at the parallel fiber-Purkinje cell synapse. Because the enhancement of the compound postsynaptic current most likely represents an increased probability of release at single synapses, and because PPF also depends on release probability, we expected that TEA would replicate the altered PPF of DKO mice when applied to slices prepared from WT mice. Indeed, application of TEA reduced PPF in WT at concentrations in agreement with the known effects of TEA on heterologously expressed Kv3.1 and

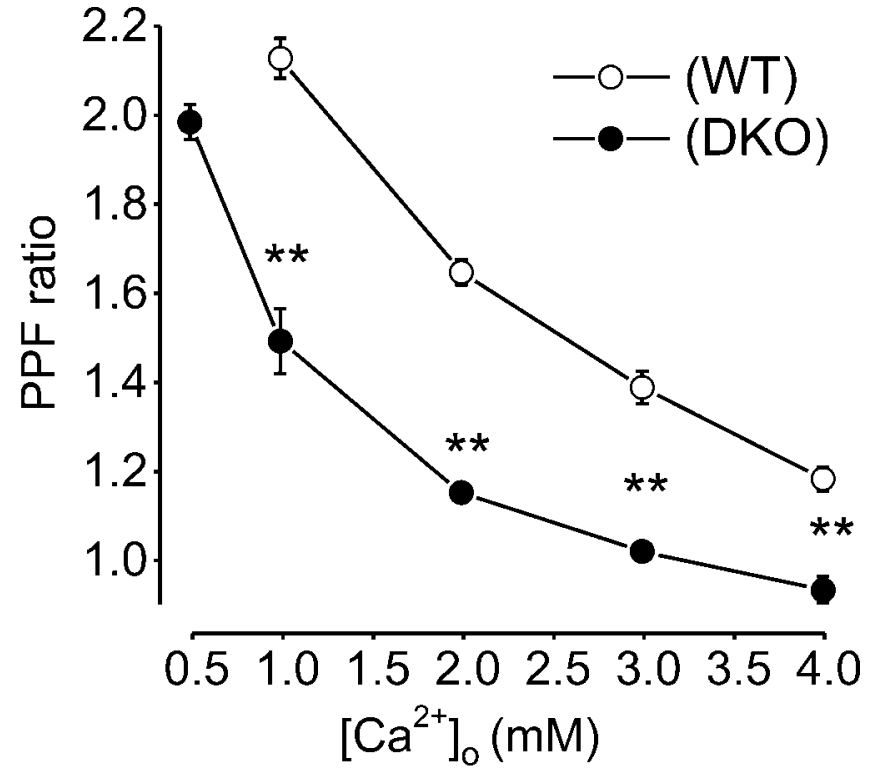

Figure 5. Reduced PPF of DKO mice can be reversed by lowering extracellular $\mathrm{Ca}^{2+}$ concentration. PPF was measured at an interval of 50 msec in the presence of different concentrations of extracellular $\mathrm{Ca}^{2+}$ in wild-type and DKO mice. Each point is the mean \pm SEM of separate experiments in four WT mice and four DKO mice. Asterisks indicate significant differences ( $p<$ 0.01) from WT. PPF ratios atdifferent interpulse intervals are shown in Table 1

Kv3.3 channels (Fig. 6) (Rudy and McBain, 2001). However, the reduction in PPF observed with application of TEA at concentrations of up to $1 \mathrm{~mm}$ was less dramatic than that seen in DKO slices. Thus, TEA qualitatively but not quantitatively replicated the DKO phenotype. Moreover, at the shortest paired-pulse interval ( $50 \mathrm{msec}$ ), PPF appeared to be slightly affected by TEA also in DKO mice, suggesting the presence of other TEA-sensitive mechanisms that affect PPF at the parallel fiber-Purkinje cell synapse.

Facilitated induction of slow metabotropic glutamate receptor type 1-mediated EPSCs in Kv3.1/Kv3.3-deficent mice The decreased paired-pulse facilitation observed in the DKO mice indicates that release probability is increased at these synapses. One consequence of increased release probability could be increased accumulation of extrasynaptic and perisynaptic glutamate during trains of parallel-fiber activity. Accumulation of perisynaptic and extrasynaptic glutamate is required for induction of the slow metabotropic glutamate receptor type 1 (mGluR1)-mediated EPSCs in Purkinje cells (Batchelor et al., 1993, 1997; Tempia et al., 1998; Brasnjo and Otis, 2001; Reichelt and Knöpfel, 2002). To investigate whether induction of slow mGluR1-mediated EPSCs is facilitated in DKO mice, trains of 1-10 stimuli were delivered to parallel fibers at $100 \mathrm{~Hz}$ in the presence of the AMPA receptor antagonist NBQX (Tempia et al., 2001; Reichelt and Knöpfel, 2002). In WT mice, clear mGluR1mediated EPSCs were observed with $\geq 3$ stimuli (Fig. 7A). Increase in the number of stimuli from 3 to 10 resulted in a close to linear increase in the amplitude of the evoked mGluR1-mediated EPSPs (Fig. 7C) (Tempia et al., 1998, 2001; Reichelt and Knöpfel, 2002). In contrast, 2 stimuli were sufficient to induce clear mGluR1-mediated EPSCs in DKO mice (Fig. $7 B$ ), and the amplitude of these EPSCs reached a maximum already at 10 stimuli (Fig. 7 C). 
Table 1. Paired-pulse facilitation at different extracellular $\mathrm{Ca}^{2+}$ concentrations in WT and DKO mice

\begin{tabular}{|c|c|c|c|c|c|c|c|c|c|}
\hline \multirow[b]{2}{*}{ Interval (msec) } & \multicolumn{4}{|l|}{ WT $\left[\mathrm{Ca}^{2+}\right]_{0}$} & \multicolumn{5}{|l|}{ DKO $\left[\mathrm{Ca}^{2+}\right]_{0}$} \\
\hline & 1 & 2 & 3 & 4 & 0.5 & 1 & 2 & 3 & 4 \\
\hline 50 & $2.13 \pm 0.05$ & $1.70 \pm 0.04$ & $1.38 \pm 0.04$ & $1.18 \pm 0.03$ & $1.98 \pm 0.04$ & $1.49 \pm 0.07$ & $1.10 \pm 0.02$ & $1.02 \pm 0.02$ & $0.93 \pm 0.03$ \\
\hline 100 & $1.77 \pm 0.09$ & $1.50 \pm 0.03$ & $1.29 \pm 0.01$ & $1.16 \pm 0.03$ & $1.72 \pm 0.07$ & $1.35 \pm 0.03$ & $1.08 \pm 0.02$ & $1.00 \pm 0.01$ & $0.96 \pm 0.02$ \\
\hline 150 & $1.61 \pm 0.07$ & $1.37 \pm 0.03$ & $1.19 \pm 0.01$ & $1.09 \pm 0.03$ & $1.47 \pm 0.04$ & $1.25 \pm 0.03$ & $1.05 \pm 0.02$ & $0.99 \pm 0.01$ & $0.97 \pm 0.02$ \\
\hline 200 & $1.43 \pm 0.07$ & $1.29 \pm 0.02$ & $1.16 \pm 0.02$ & $1.08 \pm 0.02$ & $1.40 \pm 0.04$ & $1.18 \pm 0.02$ & $1.02 \pm 0.02$ & $0.97 \pm 0.03$ & $0.95 \pm 0.02$ \\
\hline 250 & $1.31 \pm 0.03$ & $1.24 \pm 0.02$ & $1.10 \pm 0.02$ & $1.04 \pm 0.03$ & $1.32 \pm 0.08$ & $1.16 \pm 0.03$ & $1.01 \pm 0.01$ & $0.96 \pm 0.02$ & $0.94 \pm 0.04$ \\
\hline 300 & $1.26 \pm 0.03$ & $1.15 \pm 0.01$ & $1.08 \pm 0.02$ & $1.01 \pm 0.02$ & $1.17 \pm 0.08$ & $1.11 \pm 0.04$ & $0.99 \pm 0.02$ & $0.96 \pm 0.03$ & $0.95 \pm 0.01$ \\
\hline
\end{tabular}

Parallel-fiber long-term depression in Kv3.1/Kv3.3-deficient mice

Mice lacking mGluR1 exhibit a lack of long-term depression (LTD) at the parallel fiber-Purkinje cell synapses along with motor deficits (Aiba et al., 1994; Conquet et al., 1994). Because induction of the mGluR1-mediated EPSC was facilitated in DKO mice, we investigated whether these mice exhibited an alteration of LTD. In a first set of experiments we tested the effect of pairing parallel-fiber stimulation with climbing-fiber stimulation at $1 \mathrm{~Hz}$ for 5 min. This pairing protocol induced LTD (here defined as a long-lasting decrease of EPSC amplitudes to 90\%, or less, of baseline values) in 3 out 6 Purkinje cells from WT mice and in 5 of 10 cells from DKO mice (Fig. $8 A, B$ ). These results indicate that LTD can be induced in DKO mice. Next, we investigated whether induction of LTD is facilitated in DKO mice. For that, we used a protocol that involved pairing of high-frequency parallelfiber stimulation (five pulses at $100 \mathrm{~Hz}$ ) with direct depolarization of Purkinje cells (to $0 \mathrm{mV}$ for $50 \mathrm{msec} ; 60$ pairings at $0.5 \mathrm{~Hz}$ ). Pairing of tetanic parallel-fiber stimulation with direct depolarization of Purkinje cells did induce a long-term enhancement of parallel fiber-Purkinje cell transmission in WT mice ( 4 of 4 cells tested), but produced LTD in DKO mice in 3 of 4 cells tested (Fig. $8 D$ ).

\section{Discussion}

The progressive reduction in functional Kv3.1 and Kv3.3 potassium channel alleles led to progressive gait changes that ultimately resulted in severe ataxia in $\mathrm{Kv} 3.1 / \mathrm{Kv} 3.3$-deficient mice. Motor dysfunctions that result in ataxia are often associated with alterations of cerebellar function. Both Kv3.1 and Kv3.3 channels are expressed in cerebellar granule cells, suggesting that the altered firing properties of the parallel-fiber system may be involved in the observed ataxia. Hence, we investigated several presynaptic mechanisms of the parallel fiber-Purkinje cell system. We found that AP width increased, PPF decreased, and the threshold for induction of a slow mGluR-mediated EPSC decreased in $\mathrm{Kv} 3.1 / \mathrm{Kv} 3.3$-deficient mice. These alterations were allele-dose dependent. We will first discuss the physiological alterations observed in the mutant mice and then the possible relation between the altered physiology and behavior.

Altered dynamics at the parallel fiber-Purkinje cell synapse in mice lacking Kv3.1 and Kv3.3 alleles

We found that lack of Kv3.1 or Kv3.3 channels broadens the parallel-fiber AP. The observed AP width in wild-type mice is smaller than that obtained previously with similar methods (Sabatini et al., 1997) but similar to values recorded from the granule

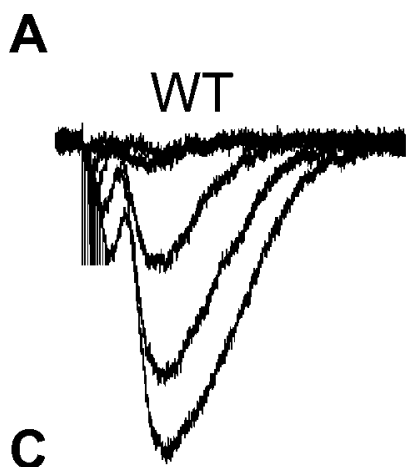

B
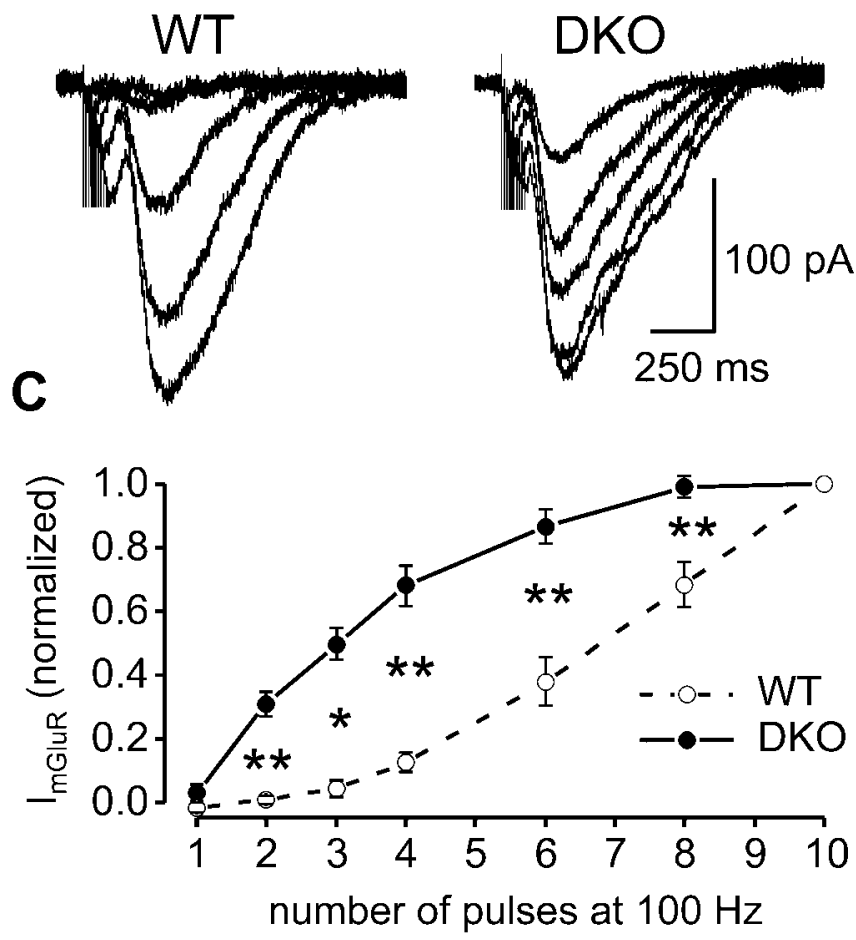

Figure 7. Facilitated induction of metabotropic glutamate receptor-mediated slow EPSCs. $A, B$, mGluR-mediated EPSCs evoked in Purkinje cells by trains of $2,4,6,8$, and 10 pulses (at 100 $\mathrm{Hz}$ ) in a WT and a DKO mouse. C, Amplitudes of mGluR-mediated EPSCs versus number of pulses. Each data point represents the mean \pm SEM ( 7 cells from 4 WT mice and 18 cells from 6 DKO mice) normalized to the response evoked by a train of 10 stimuli. Asterisks indicate significant differences $\left({ }^{*} p<0.005 ;{ }^{* *} p<0.001\right)$ from WT. 
A
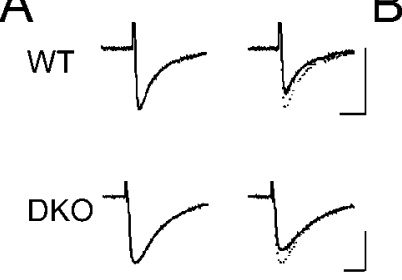

C
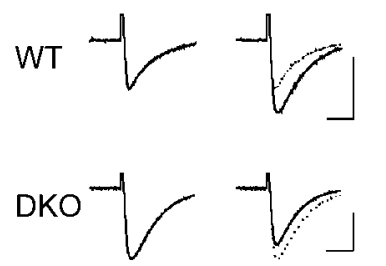

D
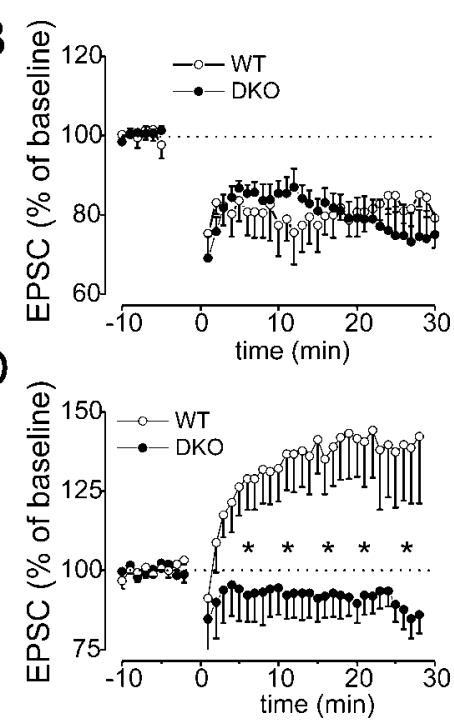

Figure 8. Long-term depression in WT and DKO mice. A, B, Pairing of parallel-fiber and climbing-fiber stimulation depressed the EPSCs elicited by parallel-fiber stimulation in WT and DKO mice. A, Records from single experiments. Left column, Baseline EPSCs. Right column, EPSCS 20 min after application of pairing protocol (dotted line indicates baseline EPSC). Calibration: $15 \mathrm{msec}, 500 \mathrm{pA}$. B, Time course of EPSC amplitudes (mean \pm SEM) of 3 of 6 and 5 of 10 experiments in which LTD (long-lasting decrease of EPSC to $<90 \%$ of control) was induced in Purkinje cells of WT and DKO mice, respectively. C, D, Pairing of high-frequency (5 pulses at 100 $\mathrm{Hz}$ ) parallel-fiber stimulation with Purkinje cell depolarization (to $0 \mathrm{mV}$ for $50 \mathrm{msec}$ ) increased EPSC s of Purkinje cells of WT mice and depressed EPSC s of Purkinje cells of DKO mice. C, Records from single experiments. Calibration: $15 \mathrm{msec}, 500 \mathrm{pA}$. D, Time course of EPSC amplitudes (mean \pm SEM) for all of the experiments (4 of 4 ) in Purkinje cells of WT mice and all of the experiments (4 of 4 ) in Purkinje cells of DKO mice. Asterisks indicate significant differences $(p<0.05)$ from WT. Error bars indicate \pm SEM, and dotted horizontal lines indicate baseline values (100\%).

cell soma using patch-clamp electrophysiology (D'Angelo et al., 1998). The ability to repetitively fire APs was only impaired at a frequency as high as $200 \mathrm{~Hz}$. The relatively modest changes in AP shape and sustained high-frequency AP firing in DKO mice indicate that other types of potassium channels such as members of the Kv1 family (Chung et al., 2001) contribute to AP repolarization in parallel fibers. Importantly, even a slight broadening of the presynaptic AP (by application of the potassium channel blocker TEA) increases presynaptic calcium influx and, in turn, leads to greatly enhanced neurotransmitter release at the parallel fiber-Purkinje cell synapse (Sabatini and Regehr, 1997). At this synapse, the relationship between AP width and presynaptic calcium influx is approximately linear; however, the relationship between AP width and compound EPSC amplitude (i.e., release probability at single release sites) follows a power law with an exponent of $n=3.1$ (Sabatini and Regehr, 1997).

In line with this pharmacological evidence, we found a dramatic alteration of PPF in Kv3.1/Kv3.3-deficient mice. A small yet significant reduction of PPF was already seen when one of the four Kv3.1/Kv3.3 alleles was absent. Kv3.1 and Kv3.3 channels have virtually indistinguishable activation and deactivation kinetics and can form heteromultimeric channels that again are very similar with respect to these properties, and the differences in inactivation properties between Kv3.1 and Kv3.3 channels may not be physiologically relevant for AP repolarization (Rudy and McBain, 2001). In agreement with the properties described in heterologous expression systems, we found that DHET and Kv3.1 single mutants show a similar reduction of paired-pulse facilitation. We therefore conclude that $\mathrm{Kv} 3.1$ and $\mathrm{Kv} 3.3 \mathrm{~K}^{+}$channels

are, with respect to AP repolarization, functionally redundant in parallel fibers. With respect to regulation by second messenger signaling system, Kv3.1 and $\mathrm{Kv} 3.3 \mathrm{~K}^{+}$channels may not be redundant even in cerebellar parallel fibers.

We found that the relationship between PPF and $\left[\mathrm{Ca}^{2+}\right]_{\mathrm{o}}$ is altered in DKO mice. Comparable PPF values were obtained at lower $\left[\mathrm{Ca}^{2+}\right]_{\mathrm{o}}$ in DKO than in WT mice. This result suggests that the decreased PPF in the mutant mice can, at least in part, be accounted for by increased presynaptic calcium influx (Zucker and Regehr, 2002). Interestingly, the effect of TEA at up to $1 \mathrm{mM}$ was less dramatic than the changes observed in Kv3.1/Kv3.3deficient mice. We can exclude neither the effects of TEA in addition to blocking Kv3.1 and $\mathrm{Kv} 3.3 \mathrm{~K}^{+}$channels of parallel fibers nor the incomplete block of native $\mathrm{Kv} 3.1 / \mathrm{Kv} 3.3 \mathrm{~K}^{+}$channels by 1 mM TEA, but it appears that the alterations of parallel fiberPurkinje cell transmission in DKO mice are not completely explained by the residual calcium hypothesis. At high $\left[\mathrm{Ca}^{2+}\right]_{\mathrm{o}}$, DKO mice exhibit paired-pulse depression (PPD) rather than facilitation (Fig. 5, Table 1). PPD has been proposed to reflect a different activity-dependent modulation of release probability, such as depletion of releasable vesicles or inhibitory actions mediated by autoreceptors (Zucker and Regehr, 2002).

\section{Facilitated induction of mGluR-mediated EPSC and LTD}

Single parallel-fiber APs produce fast, AMPA receptor-mediated EPSCs, whereas short high-frequency bursts of parallel fibers induce slow, mGluR1-mediated EPSCs in Purkinje cells (Batchelor and Garthwaite, 1993; Tempia et al., 1998). It has been demonstrated that repetitive glutamate release at parallel-fiber synapses is required for glutamate to overcome uptake and to spill over into the perisynaptic and extrasynaptic space in which it activates mGluR1 (Brasnjo and Otis, 2001; Reichelt and Knöpfel, 2002). The facilitated induction of the mGluR-mediated EPSCs in DKO mice indicates coincident activation of neighboring synapses, which occurs more frequently with increased release probability, by local accumulation of glutamate and activation of mGluR1.

One possible consequence of the facilitated activation of mGluR1 could be altered parallel-fiber LTD, because induction of parallel-fiber LTD requires the activation of mGluR1 (Aiba et al., 1994; Conquet et al., 1994). In principle, LTD could be occluded in DKO mice because of saturation of the LTD mechanism before the slice experiment or could be absent because of compensatory mechanisms. We found, however, that parallelfiber LTD can be induced in DKO mice, demonstrating that LTD is neither absent nor fully executed at the time of the slice experiment. With a pairing protocol that involves induction of an mGluR1-mediated EPSC, LTD was observed in DKO mice but not in WT mice. Interestingly, pairing high-frequency parallelfiber stimulation with direct Purkinje cell depolarization consistently produced LTP in WT mice recorded with $\mathrm{K}^{+}$-based electrodes in contrast to the previously reported modest degrees of LTD observed in $\mathrm{Cs}^{+}$-loaded Purkinje cells (Brasnjo and Otis, 2001). The LTP observed in WT Purkinje cells might be related to the form of LTP described by Tsien and coworkers (Lev-Ram et al., 2002), and our observations suggest that activity of mGluR1 can counteract this form of LTP with a resulting LTD in DKO mice.

\section{Correlation between cellular and behavioral phenotype}

What might be the possible link between the observed changes in the parallel fiber-Purkinje cell system and the ataxic phenotype of Kv3.1/Kv3.3-mutant mice? Because Kv3.1 and Kv3.3 channels are coexpressed in several neuronal subpopulations that are in- 
volved in motor coordination, we can only speculate about the causal relationship between the lack of these channels in parallel fibers and the ataxic phenotype. Mutant mice that lack parallelfiber LTD generally also exhibit an ataxic phenotype (Ito, 2001). LTD can, however, be induced in the ataxic DKO mice, ruling out this simple explanation. Moreover, along with the facilitated induction of a slow mGluR1-mediated EPSC, LTD was more readily induced in DKO than in WT mice. Whether this facilitated mGluR1 activation and LTD induction causes the motor phenotype is unclear. The behavioral consequence of the alteration in PPF at the parallel fiber-Purkinje synapse is similarly unclear. Interestingly, however, mice that lack the mGluR4 metabotropic glutamate receptor or the synaptic protein $M u n c 13$ exhibit an alteration of PPF at parallel fiber-Purkinje cell synapses along with deficient motor performance (Pekhletski et al., 1996; Augustin et al., 2001).

\section{References}

Aiba A, Kano M, Chen C, Stanton ME, Fox GD, Herrup K, Zwingman TA, Tonegawa S (1994) Deficient cerebellar long-term depression and impaired motor learning in mGluR1 mutant mice. Cell 79:377-388.

Augustin I, Korte S, Rickmann M, Kretzschmar HA, Sudhof TC, Herms JW, Brose N (2001) The cerebellum-specific Munc13 isoform Munc13-3 regulates cerebellar synaptic transmission and motor learning in mice. J Neurosci 21:10-17.

Batchelor AM, Garthwaite J (1993) Novel synaptic potentials in cerebellar Purkinje cells: probable mediation by metabotropic glutamate receptors. Neuropharmacology 32:11-20.

Batchelor AM, Knöpfel T, Gasparini F, Garthwaite J (1997) Pharmacological characterization of synaptic transmission through mGluRs in rat cerebellar slices. Neuropharmacology 36:401-403.

Brasnjo G, Otis TS (2001) Neuronal glutamate transporters control activation of postsynaptic metabotropic glutamate receptors and influence cerebellar long-term depression. Neuron 31:607-616.

Chan E (1997) Regulation and function of Kv3.3. PhD thesis, The Rockefeller University.

Chung YH, Shin C, Kim MJ, Lee BK, Cha CI (2001) Immunohistochemical study on the distribution of six members of the Kv1 channel subunits in the rat cerebellum. Brain Res 895:173-177.

Conquet F, Bashir ZI, Davies CH, Daniel H, Ferraguti F, Bordi F, Franz-Bacon K, Reggiani A, Matarese V, Conde F (1994) Motor deficit and impairment of synaptic plasticity in mice lacking mGluR1. Nature 372:237-243.

D’Angelo E, De Filippi G, Rossi P, Taglietti V (1998) Ionic mechanism of electroresponsiveness in cerebellar granule cells implicates the action of a persistent sodium current. J Neurophysiol 80:493-503.

Espinosa F, McMahon A, Chan E, Wang S, Ho CS, Heintz N, Joho RH (2001) Alcohol hypersensitivity, increased locomotion, and spontaneous myoclonus in mice lacking the potassium channels Kv3.1 and Kv3.3. J Neurosci 21:6657-6665.

Fowler SC, Birkestrand BR, Chen R, Moss SJ, Vorontsova E, Wang G, Zarcone TJ (2001) A force-plate actometer for quantitating rodent behaviors: illustrative data on locomotion, rotation, spatial patterning, stereotypies, and tremor. J Neurosci Methods 107:107-124.
Grigg JJ, Brew HM, Tempel BL (2000) Differential expression of voltagegated potassium channel genes in auditory nuclei of the mouse brainstem. Hear Res 140:77-90.

Ho CS, Grange RW, Joho RH (1997) Pleiotropic effects of a disrupted K ${ }^{+}$ channel gene: reduced body weight, impaired motor skill and muscle contraction, but no seizures. Proc Natl Acad Sci USA 94:1533-1538.

Ito M (2001) Cerebellar long-term depression: characterization, signal transduction, and functional roles. Physiol Rev 81:1143-1195.

Lev-Ram V, Wong ST, Storm DR, Tsien RY (2002) A new form of cerebellar long-term potentiation is postsynaptic and depends on nitric oxide but not cAMP. Proc Natl Acad Sci USA 99:8389-8393.

Li W, Kaczmarek LK, Perney TM (2001) Localization of two high-threshold potassium channel subunits in the rat central auditory system. J Comp Neurol 437:196-218.

Macica CM, von Hehn CAA, Yang-Wang L, Yokoyama S, Ho CS, Joho RH, Kaczmarek L (2003) Modulation of the Kv3.1b potassium channel isoform adjusts the fidelity of the firing pattern of auditory neurons. J Neurosci 23:1133-1141.

Malenka RC, Kocsis JD, Ransom BR, Waxman SG (1981) Modulation of parallel fiber excitability by postsynaptically mediated changes in extracellular potassium. Science 214:339-341.

Pekhletski R, Gerlai R, Overstreet LS, Huang XP, Agopyan N, Slater NT, Abramow-Newerly W, Roder JC, Hampson DR (1996) Impaired cerebellar synaptic plasticity and motor performance in mice lacking the mGluR4 subtype of metabotropic glutamate receptor. J Neurosci 16:6364-6373.

Porcello DM, Ho CS, Joho RH, Huguenard JR (2002) Resilient RTN fast spiking in Kv3.1 null mice suggests redundancy in the action potential repolarization mechanism. J Neurophysiol 87:1303-1310.

Reichelt W, Knöpfel T (2002) Glutamate uptake controls expression of a slow postsynaptic current mediated by mGluRs in cerebellar Purkinje cells. J Neurophysiol 87:1974-1980.

Rudy B, McBain CJ (2001) Kv3 channels: voltage-gated $\mathrm{K}^{+}$channels designed for high-frequency repetitive firing. Trends Neurosci 24:517-526.

Sabatini BL, Regehr WG (1997) Control of neurotransmitter release by presynaptic waveform at the granule cell to Purkinje cell synapse. J Neurosci 17:3425-3435.

Sanchez JA, Ho CS, Vaughan DM, Garcia MC, Grange RW, Joho RH (2000) Muscle and motor-skill dysfunction in a $\mathrm{K}^{+}$channel-deficient mouse are not due to altered muscle excitability or fiber type but depend on the genetic background. Pflügers Arch 440:34-41.

Tempia F, Miniaci MC, Anchisi D, Strata P (1998) Postsynaptic current mediated by metabotropic glutamate receptors in cerebellar Purkinje cells. J Neurophysiol 80:520-528.

Tempia F, Alojado ME, Strata P, Knöpfel T (2001) Characterization of the mGluR $_{1}$-mediated electrical and calcium signaling in Purkinje cells of mouse cerebellar slices. J Neurophysiol 86:1389-1397.

Vranesic I, Iijima T, Ichikawa M, Matsumoto G, Knöpfel T (1994) Signal transmission in the parallel fiber-Purkinje cell system visualized by highresolution imaging. Proc Natl Acad Sci USA 91:13014-13017.

Weiser M, Vega-Saenz de Miera E, Kentros C, Moreno H, Franzen L, Hillman D, Baker H, Rudy B (1994) Differential expression of Shaw-related $\mathrm{K}^{+}$ channels in the rat central nervous system. J Neurosci 14:949-972.

Zucker RS, Regehr WG (2002) Short-term synaptic plasticity. Annu Rev Physiol 64:355-405. 